\title{
S UMMAR Y
}

The modern history of the Catholic Press in Germany begins in 1821, with the establishment of the newspaper ,Der Katholik' in Mainz. Further development occured in steps. In this process phases of discussion preceded phases of practical activity (formation of newspapers or magazines). Discussions as well as special activities resulted from certain social challenges, the most known and most important being the ,Kulturkampf', the quarrel between the Prussian State and the Catholic Church (ca. 1871-1880). Strong political pressure usually resulted in expansion of the Catholic Press or - as under the Hitler regime - in the strengthening of certain structures. Periods in which there is scope for social freedom or in which there are only internal social challenges, provoked inter-catholic debates about the Catholic Press. In these debates the emphasis has been on the dissatisfaction expressed by catholic critics with the press of their own confession.

\section{RESUMEN}

Con la fundación de la revista "Der Katholik“ en Maguncia el ano 1821 comienza la historia de la prensa católica de Alemania en el sentido moderno. El desarrollo posterior se realiza en etapas en las que períodos de discusión preceden a períodos de actividad práctica (fundación de diarios o de revistas). Incentivo para la discusión, pero también para una actividad especial, han sido determinadas provocaciónes sociales de las cuales la más conocida e importante ha sido el "Kulturkampf“, la controversia del estado prusiano con la Iglesia Católica (1871-1880).

Fuertes presiones políticas produjeron generalmente la expansión de la prensa católica o - como en el caso del régimen nacional socialista - la consolidación de determinadas estructuras. Tiempos de una mayor libertad social o de mero desafío interior a la sociedad produjeron discusiones en el interior del catolicismo sobre la prensa católica. En ellas la característica es el descontento de los críticos católicos con la prensa de la propia confesión.

\section{CDU-nahe Lizenzzeitungen (VIII): „Hannoversche Neueste Nachrichten"}

\author{
von Heinz-Dietrich Fischer
}

Schon vor dem Zweiten Weltkrieg zählte Hannover zu jenen deutschen Städten, in denen sich das Pressewesen reichhaltig entfaltet hatte. ${ }^{1} \mathrm{Zu}$ den traditionsreichsten Blättern gehörten das parteilose "Hannoversche Tageblatt" (gegr. 1851), der liberale "Hannoversche Kurier" (gegr. 1854; 1940 mit dem "Tageblatt" fusioniert) sowie der parteilose „Hannoversche Anzeiger" (gegr. 1893), während die „Niedersächsische Tageszeitung " als NSDAP-Sprachrohr erst später hinzugekommen war. ${ }^{2}$ Nach dem Kriege bildeten zunächst - wie überall - Informationsblätter der Besatzungsmacht die einzigen gedruckten Nachrichtensammlungen. Im Gegensatz zu den übrigen Teilen des britischen Hoheitsbereiches ging man in dem neugeschaffenen

Dr. Heinz Dietrich Fischer ist Wiss. Assistent an der Sektion für Publizistik und Kommunikation der Ruhr-Universität Bochum. 
Land Niedersachsen erst im Sommer/Herbst 1946 dazu über, Lizenzen an Deutsche zu vergeben.

Recht eigentümliche Vorstellungen sowie eine gewisse Anlehnung an das in der amerikanischen Zone durchgeführte Prinzip der Gruppenzeitungen führten schließlich dazu, daß die erste in der niedersächsischen Landeshauptstadt Hannover lizenzierte Zeitung ein Gemeinschaftsblatt dreier Parteien wurde. Unter der ZulassungsNr. 71 der Militärregierung erschien am 3. Juli 1946 zum ersten Male die Zeitung "Hannoversche Neueste Nachrichten" als Blatt von CDU, FDP und NLP (Niedersächsische Landespartei, Vorgängerin der Deutschen Partei), wie dies aus einer Notiz in dem Blatt selber deutlich wurde. In dem Programm der „Hannoverschen Neuesten Nachrichten" hieß es u. a.: ${ }^{3}$ "... Die ,Hannoverschen Neuesten Nachrichten', die als erste durch die Militärregierung lizenzierte hannoversche Zeitung unter deutscher Verwaltung nunmehr an jedem Mittwoch und Sonnabend in den frühen Morgenstunden hinausgehen werden in die Regierungsbezirke Hannover und Hildesheim, in Teile der Regierungsbezirke Lüneburg und Stade, nach SchaumburgLippe und in die Grafschaft Schaumburg, dürfen über... Dinge offen sprechen, ohne in den Verdacht zu kommen, für sich selbst zu reden; denn sie sind nicht das Blatt irgendeiner Partei, sondern die unabhängige Gemeinschaftszeitung der CDU, der FDP und der NLP. Sie können darum der ehrliche Makler zwischen den Parteien und jenen deutschen Staatsbügern sein, die von Parteien nichts mehr wissen wollen...

In einer gemeinsamen Erklärung über die politische Ausgangssituation, welche die Zeitung zum Zeitpunkt ihrer Begründung vorfand, äußerten sich die vier Lizenzträger des Blattes folgendermaßen:4 "Unter Achtung der freien Persönlichkeit des Mitmenschen, in Demut vor dem Göttlichen und mit dem klaren Bekenntnis zur sozialen Verpflichtung wollen wir die Grundlagen eines geordneten Zusammenlebens in unserem Volke schaffen helfen. Dann aber gilt es, durch politische Vernunft, geistige Weltaufgeschlossenheit und selbstlose Mitarbeit am Wiederaufbau des zerstörten Europa das Vertrauen der Welt zurïckzugewinnen, damit sie uns wieder das Maß an innerer und äußerer Freiheit gewähre, ohne das der Friede zu einem kümmerlichen Dasein verurteilt ist. Unsere Arbeit gilt einem freien geeinten deutschen Volk, aber wir wollen dabei nie vergessen, daß die Gemeinschaft der Völker unsere größere Heimat ist. Die Lizenzträger der ,Hannoverschen Neuesten Nachrichten': Rinne, Hasler, Kwiecinski, Nannen."

Ende 1946 verfügten die „Hannoverschen Neuesten Nachrichten“ über eine Auflage von 104500 pro Ausgabe. ${ }^{5} \mathrm{Zu}$ diesem Zeitpunkt hatte sich jedoch bereits gezeigt, daß der Fortbestand der Zeitung als Dreiparteien-Blatt unmöglich war. Interne Differenzen sowie der Wunsch der Parteien, jede für sich eine eigene Zeitung zu besitzen, waren der Anlaß für diese Mitteilung an die Leserschaft Ende Dezember 1946:6 "Im Rahmen der Neuordnung des Zeitungswesens in der britischen Zone wird die bisherige Gemeinschaftszeitung der CDU, FDP und NLP, ,Hannoversche Neueste Nachrichten', auf Weisung der Nachrichtenkontrolle der Militär-Regierung ab 1. Januar 1947 als eine Parteizeitung der CDU herausgegeben werden. Damit verringert sich allerdings die Auflage, so daß wir nicht mehr in der Lage sind, allen bisherigen Beziehern die ,HNN ${ }^{\star}$ weiter zu liefern. Die hierbei entstehenden Härten werden dadurch gemildert, daß in allernächster Zeit mit der Lizenzierung neuer Zeitungen für die FDP und NLP zu rechnen ist. Die Verlage werden sich dann bemühen, eine Regelung zu treffen, die im Rahmen der verfügbaren Auflagen jedem Leser die freie Wahl seines Blattes ermöglicht. Bis 
dahin müssen wir um Geduld bitten und an jeden Bezieher nochmals die Aufforderung richten, seine Zeitung möglichst allen Bewohnern in seinem Hause zum Lesen weiterzugeben..."

Nachdem im Sommer 1946 parallel zu den CDU-FDP-NLP-orientierten "Hannoverschen Neuesten Nachrichten" (gegr. 3. 7. 1946) die sozialdemokratische "Hannoversche Presse“ (gegr. 19. 7. 1946) und die kommunistische "Niedersächsische Volkszeitung" (gegr. 16. 8. 1946) als Partei-Richtungszeitungen in Hannover geschaffen worden waren, entstanden wegen der Aufsplitterung der "Hannoverschen Neuesten Nachrichten" Anfang 1947 zwei weitere Blätter: am 6. Februar erschien unter der Lizenzträgerschaft von Henri Nannen und Johannes Siepmann die FDP-nahe Zeitung "Abendpost“, und ab 25. März kam - in Celle - die "Deutsche Volkszeitung" als Organ der Deutschen Partei (DP) heraus. Im Laufe des Jahres 1947 vermochten die nunmehr allein die CDU-Richtung vertretenden „Hannoverschen Neuesten Nachrichten“, deren Lizenzträger Richard Boese, Arnold Fratzscher und Anton Storch waren, von zwei- zu dreimal wöchentlichem Erscheinen überzugehen; die Auflage, bestehend aus der Stadtausgabe sowie 12 Bezirksausgaben, betrug 128000 Exemplare. ${ }^{7}$

In der Selbstinterpretation des 1947 geschaffenen CDU-Blattes wird das mit demselben Titel ausgestattete Vorgängerorgan verschwiegen. Dies wird besonders im Januar 1948 deutlich, als man das einjährige Bestehen der Zeitung u. a. mit diesen Worten feiert: ${ }^{8}$ "Eine beglückende Schicksalsfügung hat es gewollt, daß der erste Geburtstag unserer Zeitung mit der Feier des 100. Todestages von Josef Görres zusammenfällt. Die Huldigung, die wir Redakteure der ,Hannoverschen Neuesten Nachrichten' an diesem Tage dem großen deutschen Publizisten darbringen, soll daher ein Bekenntnis zu unserem Beruf, seinen Pflichten und seinen Freuden sein [...]. Den ewigen Wahrheiten des Christentums und den aus dem göttlichen und natürlichen Recht geborenen Forderungen der demokratischen Freiheit haben wir uns von ganzem Herzen und aus ganzer Seele verschrieben. Insoweit sind wir bewußt mehr als etwa ein Nachrichtenblatt überparteilichen Charakters, denn neben der Wiedergabe der Nachricht als solcher halten wir es für unsere Pflicht, unsere Leser im Sinne ihrer, also unserer Weltanschauung, über gewisse Strömungen und Hintergründe aufzuklären, die an dem Zustandekommen des von uns objektiv gemeldeten Geschehens mitgewirkt haben... Und in dem Geiste desselben Vertrauens, der Josef Görres leitete, wollen auch wir in den , $\mathrm{HNN}^{c}$ unsere Arbeit zum Nutzen unseres Volkes und Vaterlandes weiterführen: ,Gott hat Deutschland viel und oft gezüchtigt - aber er wird nicht von ihm lassen!"

Da weder die „Hannoverschen Neuesten Nachrichten“ noch das DP-Blatt „Deutsche Volkszeitung" um die Mitte des Jahres 1949 prosperierten, gab es Gerüchte um einen Zusammenschluß beider Blätter zu einer christlich-konservativen Zeitung. Vor allem das SPD-Blatt „Hannoversche Presse“ kolportierte derartige Meldungen über eine bevorstehende Fusion. Daß diese Behauptungen nicht aus der Luft gegriffen waren, geht aus einer schließlich Ende Juli 1949 veröffentlichten gemeinsamen Erklärung des CDU- sowie des DP-Blattes hervor: "Die Tagespresse hat in den letzten Tagen verschiedene Meldungen über einen Zusammenschluß der ,Hannoverschen Neuesten Nachrichten' (der CDU nahestehend) und der ,Deutschen Volkszeitung' (der DP nahestehend) gebracht. Tatsache ist, daß Beratungen zwischen den Lizenzträgern beider Zeitungen stattgefunden haben mit dem Ziel, durch eine solche Fusion der niedersächsischen Bevölkerung eine große Tageszeitung mit klarer politischer, kultur-, wirtschafts- und sozialpolitischer Haltung zu geben, die den 
Grundanschauungen beider Parteien entspricht. Die Lizenzträger beider Zeitungen sehen in einer solchen Zusammenlegung einen notwendigen Schritt zur wirtschaftlichen Fundierung und Wahrung der Unabhängigkeit der Lizenzpresse mit Rücksicht auf das bevorstehende tägliche Erscheinen. Alle übrigen in den unterschiedlichen Meldungen enthaltenen Mutmaßungen entsprechen nicht den Tatsachen..." Trotz dieser Erklärung wurden von anderen Zeitungen $Z$ weifel an dem Fortbestehen beider Blätter geäußert, die zu einer regelrechten Pressefehde und schließlich dieser Erklärung der „Hannoverschen Neuesten Nachrichten“ führten:10 „Werber von Konkurrenzzeitungen haben die in einzelnen Blättern veröffentlichten Meldungen über die beabsichtigte Zusammenlegung der ,Hannoverschen Neuesten Nachrichten und der ,Deutschen Volkszeitung', zu denen wir... Stellung genommen haben, $\mathrm{zu}$ einer unlauteren Bezieherwerbung benutzt. Sie behaupten, die Zeitungslieferung der Leser der ,HNN' sei ab 1. September in Frage gestellt. Um diese unwahren Werbemethoden unterbinden zu können, bitten wir unsere Leser und Austräger, uns derartige Fälle unter Angabe der Namen der Werber zu nennen, damit wir gegen die Betreffenden wegen unlauteren Wettbewerbs gerichtlich vorgehen können. Wir dürfen annehmen, daß unsere Leser diesen plumpen Versuch, mit dem sich diese Werber wieder einmal eine Provision verschaffen möchten, durchschauen. Es ist selbstverständlich, daß alle Leser der ,HNN ‘ ab 1 . September ihre Zeitung täglich erhalten, ohne daß eine Neubestellung erforderlich ist.“

Am 25. August 1949 erschien das aus der Fusion hervorgegangene Organ mit der folgenden Selbstinterpretation:11 "Der angekündigte und seit einiger Zeit von der breiten Offentlichkeit mit regem Interesse erwartete Zusammenschluß der ,Hannoverschen Neuesten Nachrichten' und der ,Deutschen Volkszeitung zu einer großen Tageszeitung für Hannover und Nordwestdeutschland wird mit dem Erscheinen dieser Ausgabe der ,Hannoverschen Allgemeinen Zeitung' vollzogen. Der Verlag freut sich, den Abonnenten beider Zeitungen versichern zu können, daß die durchgeführte Verschmelzung eine tragfähige Grundlage für eine erfolgreiche Pressearbeit im Dienste des Lesers bildet. Dabei wird es das Bestreben des Verlages und der Redaktion sein, die Bindung zwischen Leser und Zeitung weiter zu festigen und zu vertiefen. Mit der täglichen Lieferung der ,Hannoverschen Allgemeinen Zeitung' ab 1. September 1949 wird der geplante redaktionelle Ausbau in einer ständig verbesserten Unterrichtung des Lesers wirksam werden. Wie bei allen anderen Zeitungen bedingt das tägliche Erscheinen eine geringfügige Erhöhung des Bezugspreises, der indessen ein Mehrfaches an Leistung gegenüberstehen wird. Wir bitten unsere Leser und Freunde, uns ihr Vertrauen erhalten zu wollen..."

Georg Bitter urteilte 1951 über das neue Blatt:12 „Ein gewisses Kuriosum im Pressewesen der Gegenwart bildet die ,Hannoversche Allgemeine Zeitung'... Aus der gestiegenen Einflußnahme der Deutschen Partei in Niedersachsen gewinnt man ... bei der ,HAZ den Eindruck, daß der Einfluß der CDU zugunsten des Einflusses der DP weit zurückgegangen ist... Die größere Einflußnahme der Deutschen Partei auf die ,HAZ macht sich in einer steigenden publizistischen Auswertung der politischen Ziele der Partei bemerkbar... So darf man die "Hannoversche Allgemeine Zeitung' mit ihrem Einfluß von CDU und DP, besonders in dem gestiegenen Einfluß der DP, als Ausdruck eines fortschrittlichen Konservatismus bezeichnen..." Der neue Zeitungstitel war indes nicht nur als eine Kompromißformel für das Fusionsorgan der ehemaligen Blätter von CDU und DP gewählt worden, sondern bedeutete auch den Übergang der neuen Zeitung in die Hände eines bis dahin vom publizistischen Wettbewerb ausgeschlossenen Altver- 
legers, der aus der Erbmasse beider Parteizeitungen profitierte. Es handelte sich um das traditionsreiche Hannoveraner Verlagshaus August Madsack, welches zwischen 1893 und 1943 den "Hannoverschen Anzeiger" herausgebracht hatte und seither sechs Jahre vom Verlegen eines eigenen Blattes ausgeschlossen geblieben war.

Den Lesern der "Hannoverschen Allgemeinen Zeitung" blieb mithin in den Ankündigungen über das Fusionsblatt verborgen, daß faktisch weder CDU noch DP, sondern das Verlagshaus Madsack \& Co. Herausgeber der Zeitung wurde, obwohl dies aus dem Impressum unschwer zu ersehen gewesen wäre. Später hieß es aus der Sicht des Verlages über diesen Vorgang: ${ }^{13}$ „Es war vorauszusehen, daß die von der Militärregierung lizenzierten Zeitungsunternehmen ohne eigenen Druckereibetrieb in finanzielle Schwierigkeiten geraten würden, spätestens dann, wenn die Papiermarkflut vorüber war und größere Ansprüche, zum Beispiel im Hinblick auf ein tägliches Erscheinen, gestellt wurden. Das zeigte sich denn auch bald nach der Währungsreform von 1948. Die beiden für die CDU und die Deutsche Partei lizenzierten Organe, ,Hannoversche Neueste Nachrichten' und ,Deutsche Volkszeitung', von denen die erste ganz, die andere teilweise im Hochhaus an der Goseriede $^{14}$ gedruckt wurden, gerieten in eine Finanzmisere, die Verhandlungen mit dem Verlag Madsack zur Folge hatten..."

$\mathrm{Da}$ der "Hannoversche Anzeiger " 1893 als „Unparteiisches Organ für Jedermann ${ }^{\text {" }}$ gegründet worden war und in der Folgezeit stets seine politische Ungebundenheit betont hatte, war zu erwarten, daß die "Hannoversche Allgemeine Zeitung“ im Verlagshaus Madsack nicht mehr bewußt als CDU/DP-Sprachrohr apostrophiert werden würde. Und schon im Jahre 1951 wurde die unverbindlichere Richtungsbezeichnung "bürgerlich, liberal“ gewählt, bei einer Auflage von etwas über 98000 täglichen Exemplaren. ${ }^{15}$ Schon zuvor, an der Jahreswende 1950/51, hatte die „Hannoversche Allgemeine Zeitung" einen auf die Verlegertradition des Hauses Madsack hinweisenden Untertitel bekommen, worüber es in der Zeitung selbst hieß:16 "Die heutige Nummer der ,Hannoverschen Allgemeinen Zeitung' trägt zum erstenmal den so vielen unserer Leser von früher her wohlvertrauten Untertitel ,Hannoverscher Anzeiger'. Indem wir ihn im Kopfbild unserer Zeitung sichtbar in Erscheinung treten lassen, glauben wir, den Wunsch von Tausenden unserer Leser zu erfüllen, der im Lauf der letzten Jahre immer wieder an uns herangetragen wurde. Zugleich verknüpfen wir auf diese Weise die bewegte Gegenwart mit alter, hundertfach erprobter und bewährter Zeitungsüberlieferung... Unsere Zeitung wird auch weiterhin dem stetigen, durch Sachlichkeit bestimmten und von allen Sonderwünschen unabhängigen Kurse folgen, der sich über den Parteien weiß..." Diesem Fortfall jeglicher offen bekundeten politischen Parteinahme entsprach es denn auch, daß sich die Zeitung im Zeitraum 1953/54, als sie eine Auflage von 105000 aufzuweisen hatte, als "unparteiisch" in ihrer Richtungsangabe typisierte. ${ }^{17}$ Zwei Jahre später wurde schließlich sogar die Richtungsbezeichnung "unabhängig“ gewählt $1^{18}$, weitere fünf Jahre darauf dann: „Unabhängig, überparteilich“ bei einer Durchschnittsauflage von rund 130000 täglichen Exemplaren. ${ }^{19}$

Im Jahre 1968, als die "Hannoversche Allgemeine Zeitung" eine Auflage von 155000 aufwies und sich in einem Presse-Nachschlagewerk als "politisch und wirtschaftlich unabhängig“, größte Zeitung Niedersachsens", als "das Blatt mit der weitaus höchsten Leserzahl und den meisten Anzeigen in Hannover" bezeichnete20, feierte der Verlag Madsack \& Co. den 75. Geburtstag des "Hannoverschen Anzeigers", der im Untertitel der Zeitung fortlebt. Aus diesem Anlaß wurde auch eine 
etwas präzisere Formulierung der Haltung des Blattes versucht, indem es hieß:21 „Die ,Hannoversche Allgemeine Zeitung" versteht sich als eine große Regionalzeitung und ist in ihrer parteipolitisch unabhängigen Grundhaltung ein liberalkonservatives Organ. Sie will die Bevölkerung in ihrer Gesamtheit ansprechen...*

\section{Anmerkungen:}

1. Vgl. Hugo Schlüter: Die politische Tagespresse der Provinz Hannover, phil. Diss. Heidelberg 1916, sowie: Victor Wurm: Die neuere Entwicklung des deutschen Zeitungswesens unter besonderer Berücksichtigung der Verhältnisse der Provinz Hannover, phil. Diss. Heidelberg 1922.

2. Vgl. Wolfgang Hollmann: Hannover, in: Walther Heide (Hrsg.): Handbuch der Zeitungswissenschaft, 5. Lfrg., Leipzig 1941, Sp. 1624.

3. N.N.: Doch wieder Parteien?, in: „Hannoversche Neueste Nachrichten" (Hannover), 1. Jg./Nr. 1 (3. Juli 1946), S. 2, Sp. 1.

4. Daselbst, Sp. 2-3.

5. Aus: „Mitteilungen des Nordwestdeutschen Zeitungsverleger-Vereins e.V. für die britische Zone" (Düsseldorf), 1. Jg./Nr. 4, Dezember 1946.

6. An unsere Leser, in: "Hannoversche Neueste Nachrichten" (Hannover) vom 31. Dezember 1946.

7. Handbuch Deutsche Presse, hrsg. vom Nordwestdeutschen Zeitungsverleger-Verein, 1. Aufl., Bielefeld 1947, S. 247 ff.

8. Rudolf Junges: Bekenntnis zur Zeitung. Zum ersten Geburtstag der HNN, in: "Hannoversche Neueste Nachrichten" (Hannover), 29. Januar 1948.

9. Hannoversche Neueste Nachrichten/Deutsche Volkszeitung: In eigener Sache, in: "Hannoversche Neueste Nachrichten" (Hannover), 30. Juli 1949.

10. Erklärung der Verlagsleitung „Hannoversche Neueste Nachrichten“, in: „Hannoversche Neueste Nachrichten" (Hannover), 6. August 1949.

11. Verlag Hannoversche Allgemeine Zeitung: Ab heute zusammengefaßt: „Deutsche Volkszeitung"/,Hannoversche Neueste Nachrichten“, in: "Hannoversche Allgemeine Zeitung" (Hannover), 25. August 1949.

12. Georg Bitter: Zur Typologie des deutschen Zeitungswesens in der Bundesrepublik Deutschland, München 1951, S. $48 \mathrm{f}$.

13. Ernst-August Runge: In drei Stufen zum Erfolg. 75 Jahre Zeitung in Hannover, in: „Hannoversche Allgemeine Zeitung“ (Hannover), Nr. 52, 1. März 1968 (Festausgabe zum 75. Jubiläum des "Hannoverschen Anzeigers", Beilage, S. 6 [unpag.]).

14. Gemeint ist das Gebäude des Verlages Madsack, welches von den Briten den Lizenzzeitungen als Herstellungsbetrieb zugewiesen worden war.

15. Handbuch Deutsche Presse, 2. Ausgabe, Bielefeld 1951, S. 659.

16. An unsere Leser, in: „Hannoversche Allgemeine Zeitung“ (Hannover), 30./31. Dezember 1950.

17. Die Deutsche Presse 1954, Berlin 1954, S. 55.

18. Die Deutsche Presse 1956, Berlin 1956, S. 59.

19. Die Deutsche Presse 1961, Berlin 1961, S. 68.

20. Willy Stamm: Leitfaden für Presse und Werbung, 21. Ausgabe, Essen-Stadtwald 1968, S. $2 / 49$.

21. Ernst-August Runge: In drei Stufen zum Erfolg . . . a.a.a.O., S. 6, Sp. 4.

\section{S UMMAR Y}

After the Second World War licences for dailies were given as a rule only to individual parties within the British occupation zone of Germany. However, Hanover was an exception. There a paper was published for the first time on July 3, 1946 in cooperation with the three conservative parties, the Hannoversche Neueste Nachrichten. They were backed by the Christian Democratic Union (CDU) the Liberal Free Democratic Party (FDP) and the Agrarian Lower-Saxonian County Party (later German Party) DP. The 
collaboration did not last long. For from as early as January 1, 1947 each party had its own newspaper. The Hannoversche Neueste Nachrichten remained that of the CDU. After the introduction of new currency the CDU and DP papers (Deutsche Volkszeitung) merged into the Hannoversche Allgemeine Zeitung. This developed into an independent paper of conservative attitude and today has the largest circulation in Hanover.

\section{RESUMEN}

Después de la segunda guerra mundial, por regla general, en la zona de ocupación británica de Alemania se concedía la licencia de publicación sólo a diarios de determinados partidos políticos. Hubo una excepción en Hannover. Allí, el 3 de julio de 1946, apareció la primera edición de un periódico que representaba tres partidos cívicos: el „Hannoversche Neueste Nachrichten". Detrás de este diario se encontraban la Unión Democrática Cristiana (CDU), el Partido liberal Democratico Libre (FDP) y el Partido Agrario de la Baja Sajonia (el futuro partido alemán DP). Esta colaboración fracasó. Ya el primero de enero de 1947 cada partido disponía de su propio periódico. El HNN permaneció siendo el periódico de la CDU. Después de la reforma monetaria, sin embargo, los periódicos de la CDU y del DP („Deutsche Volkszeitung“) se unificaron dando origen al "Hannoversche Allgemeine Zeitung". Con el tiempo este se fue trasformando en un periódico políticamente independiente con tendencias burguesas conservadoras, que es hoy el mayor periódico de Hannover. 